\title{
Performance Degraded by the Sensor Noise at Pixel Level Image Fusion
}

\author{
Jayanth.J \\ Lecturer, ECE Department, GSSSIET \\ for Women, Mysore, Karnataka, India.
}

\author{
Dr. Shivaprakash Koliwad \\ Professor and Head, ECE Department \\ Malnad College of engineering, \\ Hassan, Karnataka, India.
}

\begin{abstract}
Remote sensing is defined as obtaining information about a Performance metrics for measuring absolute degradation and their gain in fused image quality are proposed when fusing noisy input modalities. This considers fusion of noise patterns, is also developed and used to evaluate the perceptual effect of noise corrupting homogenous image regions (i.e. areas with no salient features). These metrics are employed to compare the performance of different image fusion methodologies and feature selection/information fusion strategies operating under noisy input conditions. The aim of this paper is to define appropriate metrics which measure the effects of input sensor noise on the performance of image fusion systems.' noisy fusion" metrics are developed and used, in the first two scenarios, to measure the effects of additive sensor noise on the performance of several signal-level image fusion algorithms operating across a range of input signal-to-noise ratio (SNR) values.
\end{abstract}

\section{Keywords}

Image fusion, Fusion performance évaluation, Sensor noise effect.

\section{INTRODUCTION}

An illustration of a single sensor image fusion system is shown in Figure1. The sensor shown could be a visible-band sensor such as a digital camera. This sensor captures the real world as a sequence of images. The sequence is then fused in one single image and used either by a human operator or by a computer to do some task. For example in object detection, a human operator searches the scene to detect objects such intruders in a security area [21].

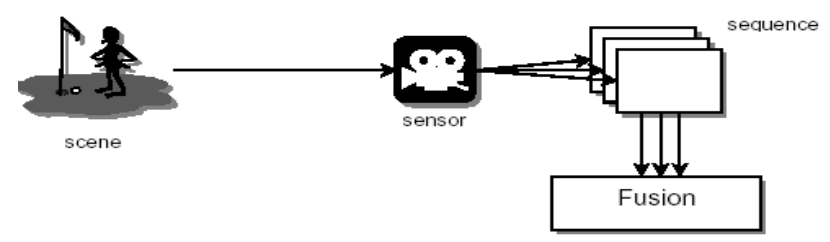

Figure 1 Single Sensor Image Fusion Systems

This kind of systems has some limitations due to the capability of the imaging sensor that is being used. The conditions under which the system can operate, the dynamic range, resolution, etc. are all limited by the capability of the sensor. For example, a visible-band sensor such as the digital camera is appropriate for a brightly illuminated environment such as daylight scenes but is not suitable for poorly illuminated situations found during night, or under adverse conditions such as in fog or rain.
A multi-sensor image fusion system overcomes the limitations of a single sensor vision system by combining the images from these sensors to form a composite image [6]. Figure 2 shows an illustration of a multi-sensor image fusion system. In this case, an infrared camera is supplementing the digital camera and their individual images are fused to obtain a fused image. This approach overcomes the problems referred to before, while the digital camera is appropriate for daylight scenes, the infrared camera is suitable in poorly illuminated ones.

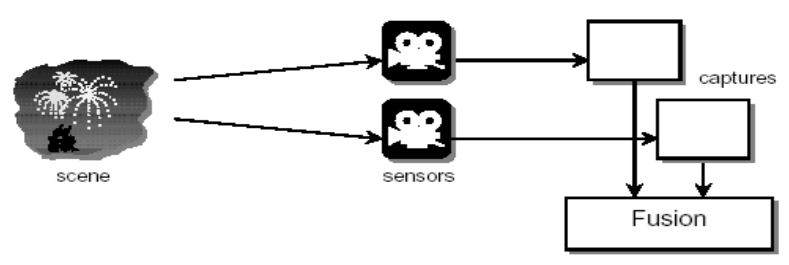

Figure 2 Multisensor Image Fusion Systems

The benefits of multi-sensor image fusion include:

- Extended range of operation - multiple sensors that operate under different operating conditions can be deployed to extend the effective range of operation. For example different sensors can be used for day/night operation.

- Extended spatial and temporal coverage - joint information from sensors that differ in spatial resolution can increase the spatial coverage. The same is true for the temporal dimension.

- Reduced uncertainty - joint information from multiple sensors can reduce the uncertainty associated with the sensing or decision process.

- Increased reliability - the fusion of multiple measurements can reduce noise and therefore improve the reliability of the measured quantity.

- Robust system performance - redundancy in multiple measurements can help in systems robustness. In case one or more sensors fail or the performance of a particular sensor deteriorates, the system can depend on the other sensors

Compact representation of information - fusion leads to compact representations. For example, in remote sensing, instead of storing imagery from several spectral bands, it is comparatively more efficient to store the fused information.

Fusion is performed in the pyramid domain by creating a fused pyramid using the information present in the input pyramids. This is usually referred to as the pyramid fusion process and can be performed in a number of different ways. The most successful approach is to use some form of feature selection that directly 
compares input pyramids coefficients on the basis of their importance and selects the one deemed more important for the fused pyramid [13]. Practically, selection maps are formed that indicate, at each fused pyramid pixel, which of the input pyramids is to be used as a source to copy the value from. These selection maps are the most optimal entry point for feature level information, explained in the following section. Finally, once a fused pyramid is completed it is input into the image reconstruction process, reverse of image decomposition, which produces the fused image.

Within these application areas, where there is a tendency to employ several sensing modalities under a wide range of operating conditions, the prospect arises of fusing input images of low visual quality. As a result, "noisy" input information associated with individual sensors may affect significantly fusion system performance. This is because input "noise" may be treated by the fusion system as valid information and transferred to the fused output image. Furthermore input image noise may affect the selection/fusion process of the MSL-IF system in a way that introduces additional unwanted artefacts and distortion into the fused image [1-6].

The performance characteristics of image fusion algorithms, operating in noise free conditions, are considered in a number of papers. Zhang and Blum [6] present a thorough investigation into several multiresolution fusion methodologies for a digital camera application. Fusion schemes are categorised according to their basic multiresolution/pyramid image representation approach and mechanisms for pyramid coefficient fusion. Pohl and van Genderen [27] provide a comprehensive review of fusion techniques as applied to the field of remote sensing.

Figure 3 show diagrammatically the fusion process the same approach is taken when both input images are corrupted images is scaled to 7 . The noisy fused output images are produced while fusing corrupted inputs at matching SNR values with a true "noise-less" fused reference image.

Multisensor imaging arrays often include (i) visible light sensors that measure scene illumination in the visible spectrum $(0.45-0.7$ $1 \mathrm{~m}$ ) (ii) infrared sensors that measure the thermal radiance of scene objects in the infrared part of the spectrum $(1.5-15 \mathrm{~lm})$ and (iii) low light or image enhanced cameras [25]. In general, sensor noise is the result of several processes associated with the underlying physics of recording an observation [8-12]. Typically however, additive noise is the predominant component of noise encountered in such devices. Additive noise is modelled as a random signal that is simply added to the original signal. In the practical model used in this investigation, an input image $\mathrm{A}$ is corrupted to yield image $A_{n}$ by (i) generating a noise-seed signal $\mathrm{N}$ according to particular sensor noise characteristics, (ii) scaling $\mathrm{N}$ by an appropriate factor $\mathrm{k}_{\mathrm{n}}$ to produce a desired signal-to-noise ratio " $n$ " and (iii) adding the

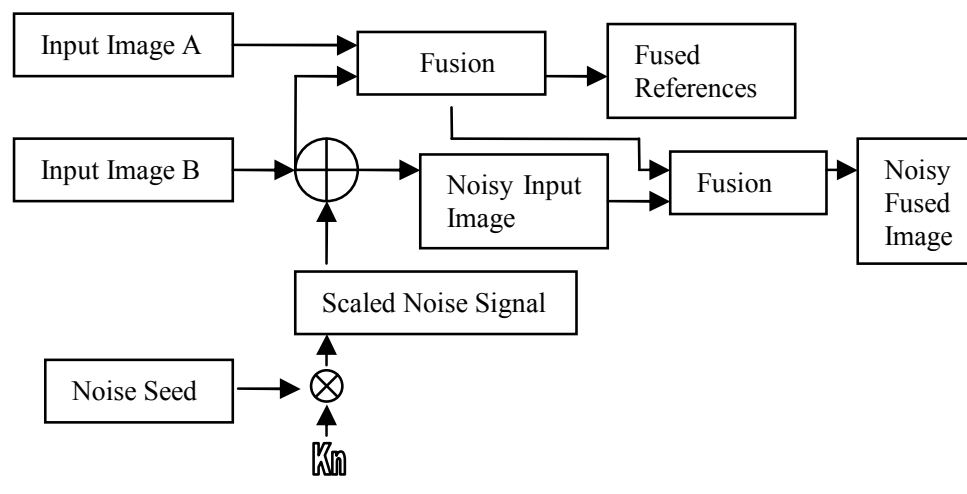

Figure 3 Single noisy input fusion and corresponding noise free fusion processes

\section{NOISY IMAGE PERFORMANCE EVALUATION}

Noise-free fusion system performance metric such as the root mean square error (RMSE), compare the output fused image with a reference image and form a distance between the two, e.g. square of the difference in the RMSE metric. These metric, which are potentially applicable to the case of noisy fusion measure "relative" fusion performance (with respect to a noise free fused reference) .

Both the "relative" and "absolute" image fusion performance of a systems operating under noisy input conditions can be objectively measured using the subjectively meaningful fusion performance evaluation. In this approach, visual information is associated with "edge" related information that is measured at each image pixel. An image fusion process that succeeds in transferring all of the visual information from any number of inputs into the fused image is said to have achieved ideal fusion.

\subsection{Overall Degradation of Performance in Noisy Image Fusion}

Main objective of an image fusion system is to transfer, the content of input images into a fused output image as faithfully as possible. This implies a possible "loss" of information as well as the introduction of "artefacts" in the fused image. With this in mind, the $\mathrm{Q}^{\mathrm{AB} / \mathrm{F}}$ p measure $[13,14]$, see Eq. (6) is a fusion performance metric whose value increases toward unity as the amount of information loss and artefacts in F decreases. However, when input images are corrupted by noise, loss of input "noise information" in the output image is an advantageous characteristic of the underlying fusion process.

Measuring only the representation of "true", scene information in the fused image solves the problem of taking into account correctly the loss of noise information. This true information is contained in the noise free input images, $\mathrm{A}$ and $\mathrm{B}$, and meaningful performance assessment is achieved using the $\mathrm{Q}^{\mathrm{AB} / \mathrm{Fn}}{ }_{\mathrm{p}}$ metric. The value of $Q^{A B / F n}{ }_{p}$ increases when the fused output image is a more accurate representation of the noise free $\mathrm{A}$ and $\mathrm{B}$ images, i.e. when there is a reduction of noise in $F n . Q^{A B / F n}$ measures the overall success, of the fusion scheme $p$, in representing true scene information in the noisy fused image Fn, and can be used to determine fusion performance under noisy input conditions. Thus 
for decreasing SNR values “' $n$ ", $Q^{\mathrm{AB} / \mathrm{Fn}}$ p describes the absolute degradation of fusion performance with increasing noise. Notice that $\mathrm{Q}^{\mathrm{AB} / \mathrm{Fn}} \mathrm{p}$ takes into account the effects of artefacts and distortions introduced by the fusion process itself.

Another metric of "relative" noisy fusion performance is the change in degradation found in the fused image with respect to that of input images. This metric considers the fact that robust fusion algorithms may suppress noise effects and, as a result, fused images may degrade less than input images for corresponding levels of noise. Conversely, fused images obtained via noise sensitive algorithms either degrades in the same manner as input images or contain amplified noise. By comparing the degradation of information in input images corrupted by noise, with that of fused images, a relative performance (information) gain achieved by the fusion process can be measured. A measure of relative fusion "gain" can be defined when one or two inputs are corrupted by noise. In particular, in the single noisy input case, $\mathrm{Q}^{\mathrm{AA} / \mathrm{An}}{ }_{\mathrm{p}}$ measures the loss of visual information as a result of corrupting the noise-free image A with $\mathrm{n} \mathrm{dB}$ noise to obtain An. Also, $Q^{\mathrm{FF} / \mathrm{Fn}}$ measures the loss of information at the output of the fusion system, due to the input being corrupted by noise. The relative effect of noise on the visual information observed in the input and output fused images can then be defined as the relative difference between the degradation of the fused image $Q^{\mathrm{FF} / \mathrm{Fn}}{ }_{p}$ and the degradation of the input image $\mathrm{Q}_{\mathrm{pA} / \mathrm{An}}^{\mathrm{A}}$. For the single noisy input case then, the relative noisy fusion gain $\mathrm{D}^{\mathrm{p}}{ }_{\mathrm{n}}$, is defined as.

$$
D=\frac{Q^{F F / F n}-Q^{A A / A n}}{Q^{A A / A n}}
$$

Most of the fusion method treats noise patterns as valid information and fuse them directly into $F_{n}$. This transfer of noise information is not desirable and for a decreasing SNR, a robust fusion method preserves the true information in the fused image and the rate of decrease of $Q^{\mathrm{FF} / F n}$ is slower than that of $Q^{\mathrm{AA} / \mathrm{An}}{ }_{p}$ resulting in a positive value of $\mathrm{D}^{\mathrm{p}}{ }_{n}$. In general, $\mathrm{D}^{\mathrm{p}}{ }_{\mathrm{n}}$ is negative when input noise is "amplified" by the fusion process, i.e. further loss of information in $\mathrm{F}_{\mathrm{n}}$ is caused by the fusion process.

\subsection{Performance Measure in Noisy Fusion}

Preservation of significant information from the uncorrupted input images into the fused images is concentrated on measuring their performance. Noise in this area may cover a large part of the fused image, has a distracting effect on the visual quality when both input images are corrupted by noise, the properties of noise patterns in the fused image depends on the fusion algorithms should discern between real information and noise.

The effect of different fusion approaches an output noise is evaluated here, while fusing signals containing only random noise patterns of the resulting fused signals. Fused noise power directly relates directly to the "detectability" of the noise pattern in the fused image. Relative change in the noise power, produced by a fusion scheme $\mathrm{p}$ is measured as the noise power gain $\mathrm{S}_{\mathrm{p}}$, defined in Eq. (9), where $\sigma_{0 \mathrm{p}}^{2}$ and $\sigma_{0 \mathrm{i}}^{2}$ are the fused and input noise variances respectively. Fusion algorithms, which amplify input noise, have $S_{p}>1$, while noise suppression in fused image produces $S_{p}<1$

$$
S_{P}=\frac{\sigma_{0}^{2} p}{\sigma_{0}^{2} i}
$$

\section{RESULTS AND DISCUSSION}

The image fusion technique for RS data GUI (Graphic User Interface) will be introduced. It was developed with the Matlab tool GUIDE (GUI Design Environment). This Toolkit intends to perform fusion of two source images using the different methods, in a user-friendly.

During the experimentation in the first a true, "noise-less" fused image is produced for each input pair, where fusion of images A and B using different methods like DWT and Laplacian pyramid. In this work we take performance under the single noisy input scenario and the same is done by taking the when both images are corrupted at different level of SNR

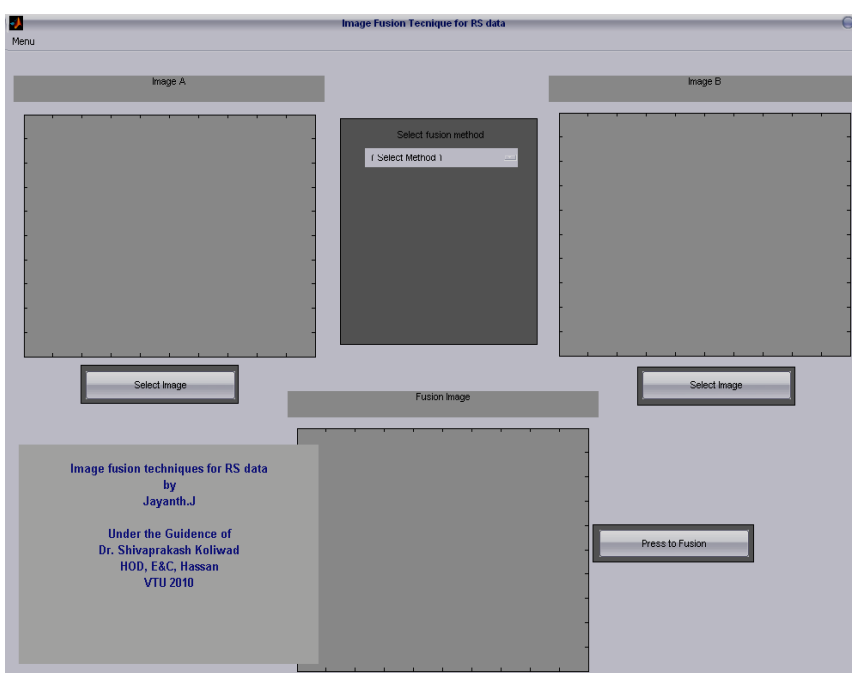

Figure 4 Snapshot of Fusion toolkits Main Screen

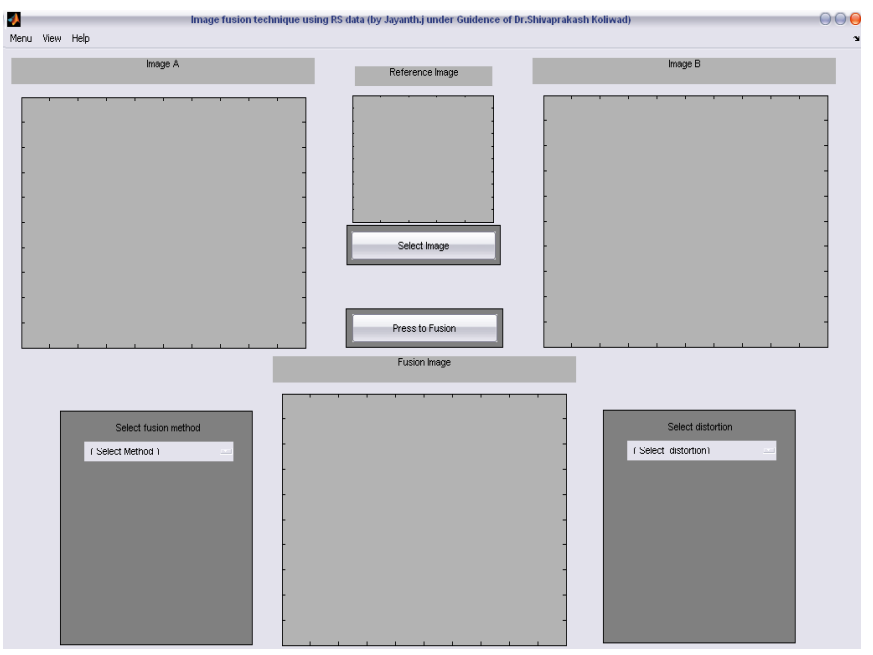

Figure 5 Snapshot for selecting the images methods and distortion 


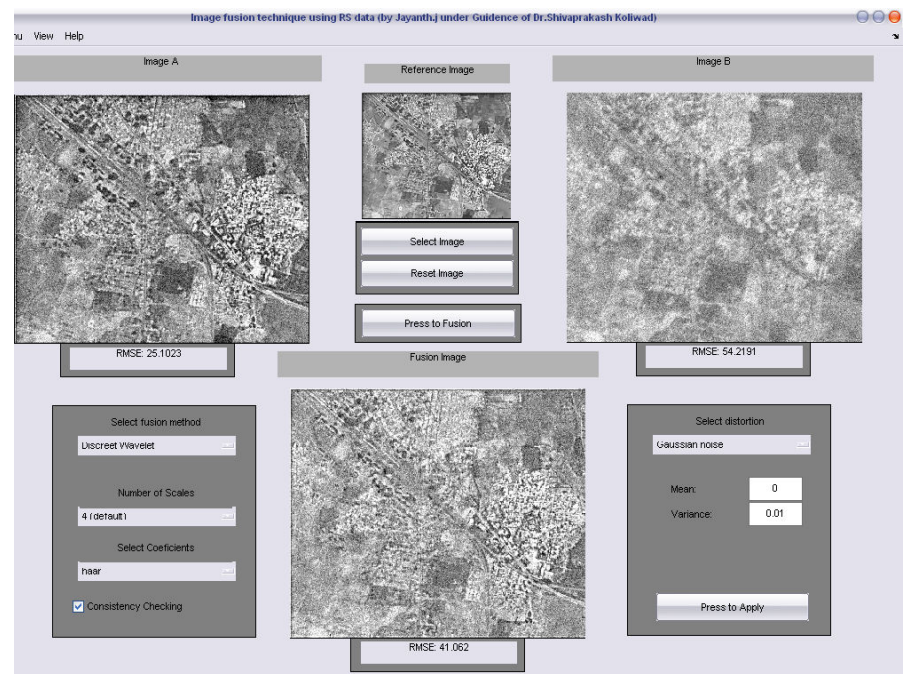

Figure 6 Snapshot for applying Gaussian noise and their RMSE value using DWT and Laplace

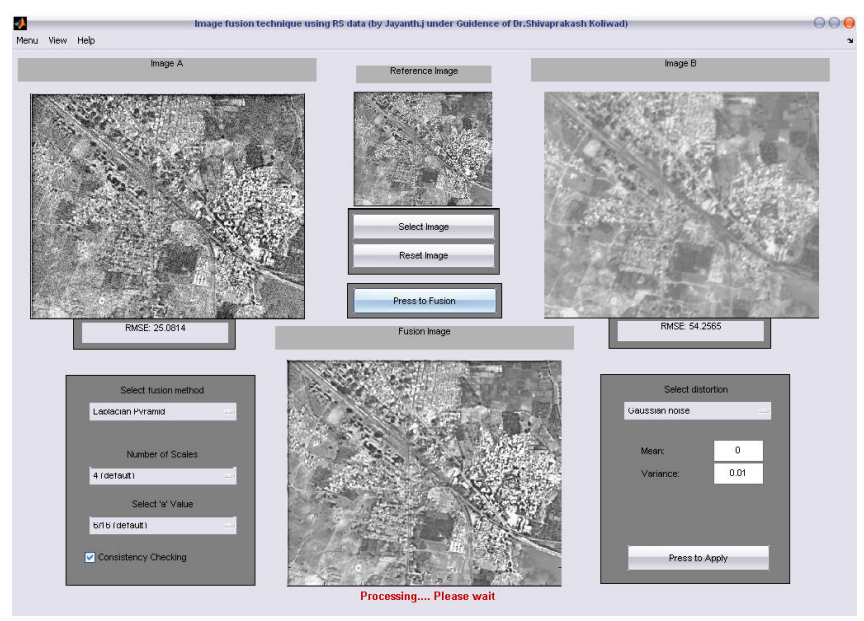

Figure 7 Snapshot for applying Gaussian noise to only one image A

\subsection{Performance of Image Fusion in the presence of Input Noise.}

In our work selection of coefficients approach is used for a feature selection mechanism. In this work images obtained from the region are decomposed into several lower resolution sub-bands that contain varying or same size information which operates the fusion process effectively at different scale range.

The Laplacian pyramid representation is formed by the expanded version which is obtained by filtering each level of the Laplace pyramid. This process decomposes for each input image according to their pyramids according to their orientation. This introduces orientation sensitivity to the fusion process whereby information of different orientation is fused independently.

The Discreet Wavelet Transform (DWT) approach decomposes an input image using a 1D and 2D filter banks, having sub-bands of images at each resolution level. The values of DWT pyramid coefficients reflect local area rather than direct pixel level at corresponding scales, the fused output images are obtained from the fusion pyramid by applying reconstruction process.

Fusion performance results are shown in Figure 8 when the above scheme operate with only one noisy input image Figure 8 displays absolute performance values for the corrupted visible light input data. The absolute performance with decreasing SNR values, a behavior that levels off below $5 \mathrm{db}$ above $10 \mathrm{db}$ the laplacian pyramid fusion performs slightly better than the DWT indicating relative insensitivity to small, random changes in the input signal. The fusion gain performance degradation results are shown in Figure 9. Image averaging is least affected by the increase in input noise, while DWT is most sensitive.

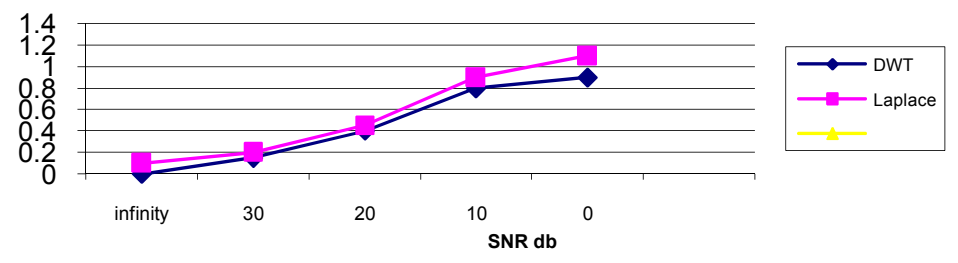

Figure 8 Single Noisy fusion Gain $\mathrm{D}^{\mathrm{P}} \mathrm{n}$ performances against input SNR for Laplacian and DWT.

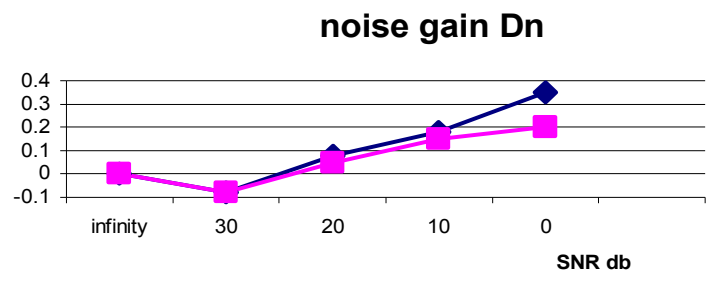

Figure 9 Fusion of two noisy inputs fusion gain $D^{P} n$ performance against SNR of Laplace and DWT.

A final comparison between these noisy performances with respect to the selection of their coefficient the and $D^{p}{ }_{n}$ capture the subjective effective of noise on "coherent" edge detection on related to true scene visual information, on the other hand, in "uniform" image regions which are subjectively less meaningful since the human visual system filters out of which people can see through noise, i.e. the presence of input noise, smaller the selection template size will preserve better true scene information. A graphic example of fusion performance of FS/FP is demonstrated in Figure 10. Noise free input images are shown in Figure 10(a) and (b) and the Corrupted SNR vales of 10dB Figure 10 (d). Direct DWT fusion scheme produced the noise free fused image as shown in Figure 10 (c), with value of $\mathrm{Q}^{\mathrm{AB} / \mathrm{Fn}}{ }_{\mathrm{p}}=0.689$ with an objective measure. When significant noise corruption is present at the input images, DWT fusion performance suffers where the main objects in the input images are hardly visible in Figure 10(e). 


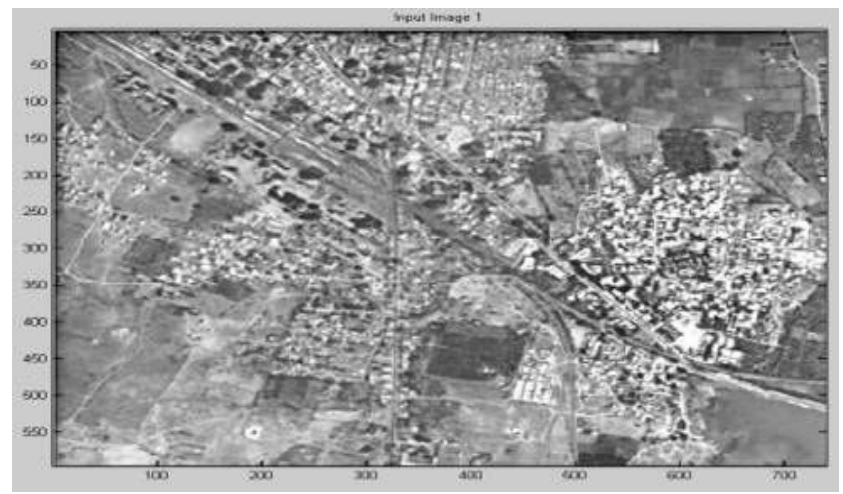

(a)

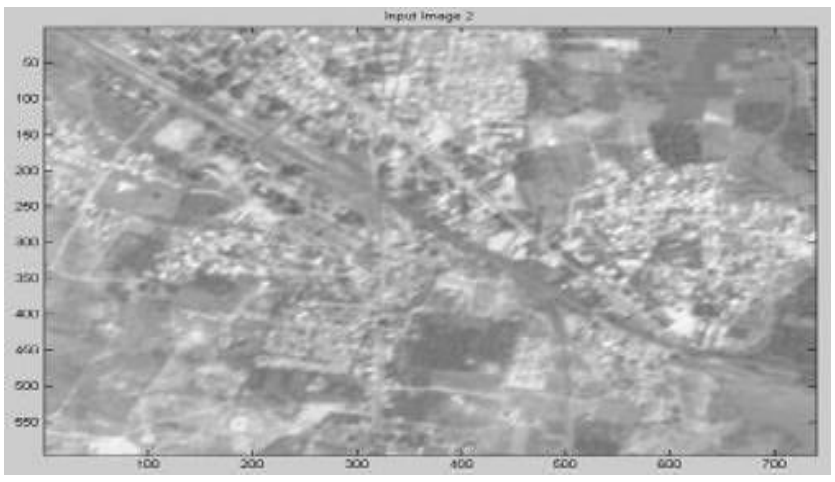

(b)

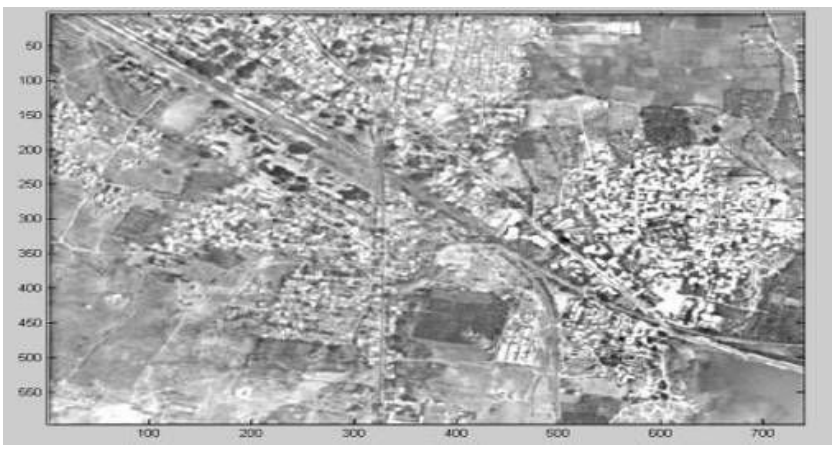

(c)

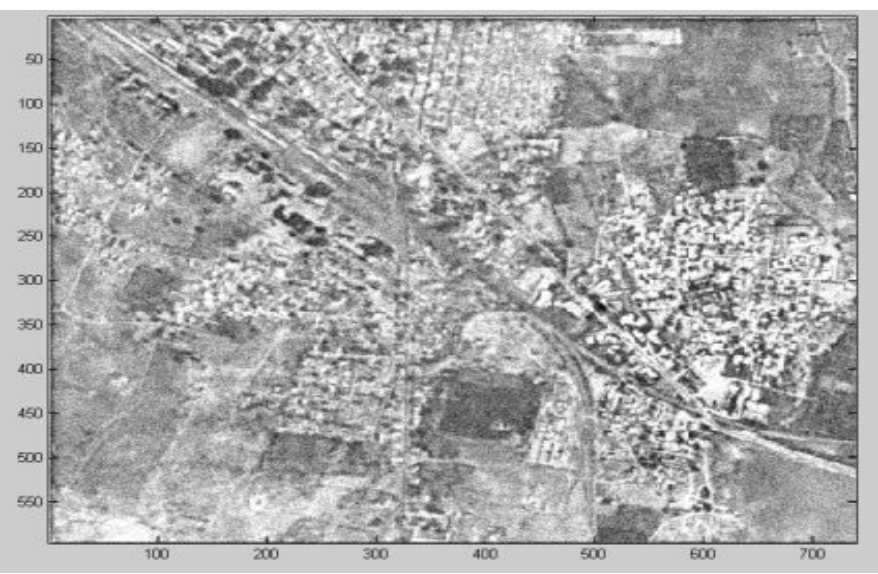

(d)

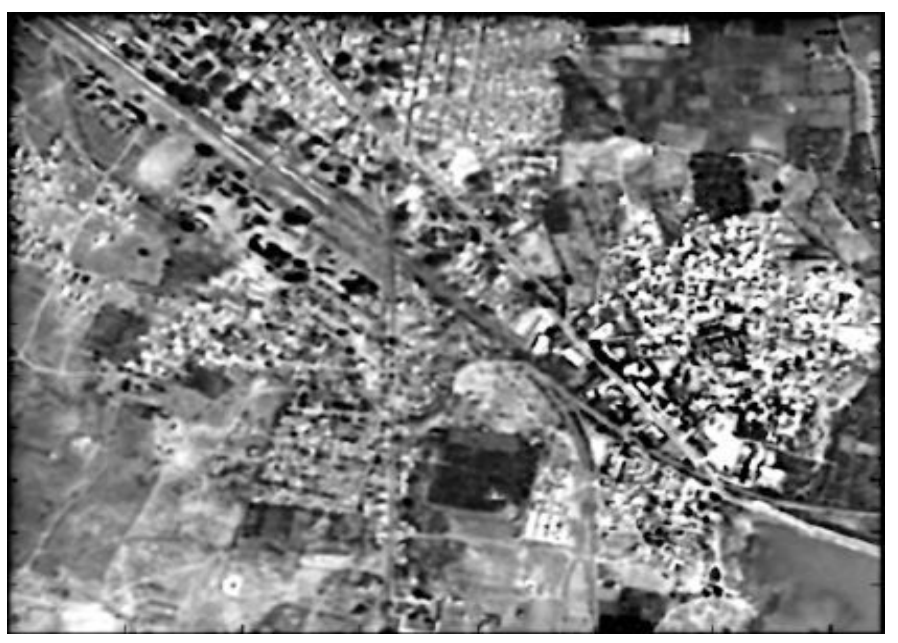

(e)

Figure 10 Input Images (a) Noise free input image A (B) Noise free Input image B (c) Noise free Fused Image (d) Noisy Fused Image at SNR 10db (e) Noisy Fused Image.

\section{CONCLUSION}

The performance of image fusion system which is significantly affected within the visible range were studied, where input signals where corrupted to check the performance of absolute fusion performance and fusion gain, were evaluated at different feature selection at multiresolution technique generally preserve or in some case even increase noise in fused signals. DWT fused images degrade least for increasing level of input noise.

In multiresolution fusion the feature selection under noisy input condition selection of coefficients were through coefficient combining method and weighting average method, provides best absolute fusion performance even though the feature selection methods exhibit performance limitation when input images are free of noise. Performance is found dependent strongly on the selection of their coefficient would better preserve the true scene information.

\section{ACKNOWLEDGMENTS}

It is a pleasure to recognize the many individual who have helped me in completing this technical paper. I sincerely express heartiest thanks to my guide Dr. Shivaprakash Koliwad for all the technical guidance, encouragement and support throughout this process.

Finally I thank to Dr. Ashok kumar, Dr. C.G. Patil and Choodarathnakar A.L for his external guidance for the analysis of the data and to our principals and my friends for their support for completing this work.

\section{REFERENCES}

[1] P. Burt, R. Kolczynski, Enhanced image capture through fusion, Proceedings of the Fourth International Conference on Computer Vision, pp. 173-182, 1993.

[2] H. Li, B. Munjanath, S. Mitra, Multisensor image fusion using the wavelet transform, Graphical Models and Image Processing 57 (3) 235-245, 1995.

[3] L. Chipman, T. Orr, L. Graham, Wavelets and image fusion, Proceedings of SPIE 2569 208-219, 1999. 
[4] L. Ramac, M. Uner, P. Varshney, M. Alford, D. Ferris, Morphological filters and wavelet based image fusion for concealed weapons detection, Proceedings of SPIE 3376, 110-119, 1998.

[5] L. Grewe, R. Brooks, Atmospheric attenuation reduction through multi-sensor fusion, Proceedings of SPIE 3376102 109, 1998.

[6] Z. Zhang, R. Blum, A categorization of multiscaledecomposition based image fusion schemes with a performance study for a digital camera application, Proceedings of the IEEE 87 (8), 1315-1326, 1999.

[7] G. Waldman, J. Wootton, Electro-optical systems performance modelling, Artech House, Norwood, 1993.

[8] A. Frenkel, M. Sartor, M. Wlodawski, Photon-noise-limited operation of intensified CCD cameras, Applied Optics 36 (22), 5288-5297, 1997.

[9] G. Healey, R. Kondepudy, Radiometric CCD camera calibration and noise estimation, IEEE Transactions of PAMI 16 (3),267-276, 1994.

[10] M. Nelson, J. Johnson, T. Lomheim, General noise processes in hybrid infrared focal plane arrays, Optical Engineering 39 (11), 1682-1700, 1991.

[11] R. Boie, I. Cox, An analysis of camera noise, IEEE Transactions of PAMI 14 (6), 671-674, 1992.

[12] A. Prabala, CCD vs. CMOS imagers for Sci/industrial cameras, Advanced Imaging (January) 16-42, 2000.

[13] C. Xydeas, V. Petrovi_c, Objective image fusion performance measure, Electronic Letters 36 (4), 308-309, 2000.

[14] C. Xydeas, V. Petrovi_c, Objective pixel-level image fusion performance measure, Proceedings of SPIE 4051, 89-99, 2000.

[15] J. Beynon, D. Lamb, Charge-Coupled Devices and their Applications, McGraw-Hill, London, 1980.
[16] A. Fairhurst, A. Lettington, Method of predicting the probability of human observers recognising targets in simulated thermal images, Optical Engineering 37 (3), 744 751,1998

[17] S. Ross, a First Course in Probability, MacMillan, New York, 1994.

[18] V. Petrovi_c, C. Xydeas, Computationally Efficient Pixellevel Image Fusion, Proceedings of Eurofusion99, Stratfordupon- Avon, pp. 177-184, October 1999.

[19] A. Toet, Hierarchical image fusion, Machine Vision and Applications 3, 3-11, 1999.

[20] A. Akerman, Pyramid techniques for multisensor fusion, Proceedings of SPIE 1828, 124-131, 1992.

[21] S. Mallat, A theory for multiresolution signal decomposition: the wavelet representation, IEEE Transactions of PAMI 11 (7), 674-693, 1999.

[22] V. Petrovi_c, C. Xydeas, Multiresolution image fusion using cross band feature selection, Proceedings of SPIE 3719, 319326,1999

[23] W. Handee, P. Wells, The Perception of Visual Information, Springer, New York, 1997.

[24] R. Sharma, M. Pavel, T. Leen, Multi-stream video fusion using local principal components analysis, Proceedings of SPIE 3436, 717-725m, 1998.

[25] M. Sonka, V. Hlavec, R. Boyle, Image Processing, Analysis and Machine Vision, PWS Publishing, Boston, 1999.

[26] C. Pohl, J. van Genderen, Multisensor image fusion in remote sensing: concepts, methods and applications, International Journal of Remote Sensing 19 (5), 823-854, 1998. 